\title{
A case of hypothyroidism mimicking acute coronary syndrome
}

\author{
Huseyin Gunduz ${ }^{1}$, Huseyin Arinc ${ }^{1}$, Mustafa Yolcu ${ }^{1}$, Ramazan Akdemir ${ }^{2}$, \\ Mustafa Kanat ${ }^{1}$ \& Cihangir Uyan ${ }^{1}$ \\ ${ }^{1}$ Department of Internal Medicine and Cardiology, Izzet Baysal Medical Faculty, Izzet Baysal University, \\ Bolu, 14100, Turkey; ${ }^{2}$ Department of Cardiology, Duzce Medical Faculty, Izzet Baysal University, Duzce, \\ Turkey
}

Received 11 May 2005; accepted in revised form 21 June 2005

Key words: acute coronary syndrome, hypothyroidism, troponin

\begin{abstract}
Hypothyroid patients have increased concentrations of creatinine kinase that is mostly due to increased CK-MM. However, CK-MB has also been reported to increase above reference values in hypothyroid patients without apparent myocardial damage. This may create confusion during the evaluation of myocardial injury in a hypothyroid patient presenting with chest pain. Troponin I is considered as a superior marker for the diagnosis of myocardial infarction in hypothyroid patients. However, there are some reports showing an increase in the level of troponin I without any myocardial damage in hypothyroid patients as in our case. In this report, we present a 47 years old male hypothyroid patient who had chest pain, abnormal electrocardiographic findings and increased cardiac enzymes suggesting acute coronary syndrome although he had normal coronary arteriogram.
\end{abstract}

Hypothyroidism has cardiovascular consequences resulting from direct influences of thyroid hormone deficiency on the heart and from adverse effects on circulating lipid concentrations and hypertension that may cause in accelerated atherosclerosis and coronary artery disease [1]. In addition, creatine kinase and troponins may increase in hypothyroid patients without apparent myocardial damage [2]. This may create confusion in evaluating myocardial injury in patients with hypothyroidism presenting with chest pain and abnormal electrocardiographic findings.

In this report, we presented a 47 years old male hypothyroid patient who had chest pain, abnormal electrocardiographic and laboratory findings suggesting acute coronary syndrome although he had normal coronary arteriogram.

\section{Case}

A 47 years old previously healthy male was admitted within an hour after onset of typical chest pain, and cold sweating. One day before admission, the patient noted the initial onset of several episodes of left-sided chest pain spreading to epigastrium and radiating into his neck and left $\mathrm{arm}$. The first pain of the patient appeared at rest, lasted approximately $30 \mathrm{~min}$, and then resolved. 
The pain was not positional and was never associated with activity.

His history revealed that effort related dyspnea (NYHA II), weakness, and palpitation for 1 year. He was smoking one pack of cigarettes daily for the last 25 years. He also had hyperlipidemia and family history of coronary artery disease.

On physical examination, his heart rate was 66 beats per minute and blood pressure 120/ $80 \mathrm{mmHg}$. There was not any murmur, friction rub or gallop on cardiac examination. His lungs were clear to auscultation, and abdominal examination findings were normal. He was in sinus rhythm with sequential $\mathrm{T}$ wave inversion in $\mathrm{V}_{2}-\mathrm{V}_{6}$ and $\mathrm{D}_{2}, \mathrm{D}_{3}$ aVF leads suggesting of ischemia on electrocardiography (Figure 1). Chest radiography was normal and transthorasic echocardiography showed minimal pericardial effusion.

Laboratory test results were as follows; creatine kinase $1164 \mathrm{IU} / 1$, creatine kinase-MB $78 \mathrm{IU} / 1$, troponin I $0.80 \mathrm{ng} / \mathrm{ml}(\leq 0.40 \mathrm{ng} / \mathrm{ml})$, and alanine aminotransferase, aspartate aminotransferase, gamma-glutamyl transferase and lactate dehydrogenase were mildly increased, total cholesterol $293 \mathrm{mg} / \mathrm{dl}$, triglycerides, $78 \mathrm{mg} / \mathrm{dl}$, HDL, $70 \mathrm{mg} /$ dl, LDL, $207 \mathrm{mg} / \mathrm{dl}$.

Acute coronary syndrome was diagnosed by enzymatic analyses and ongoing chest pain. After

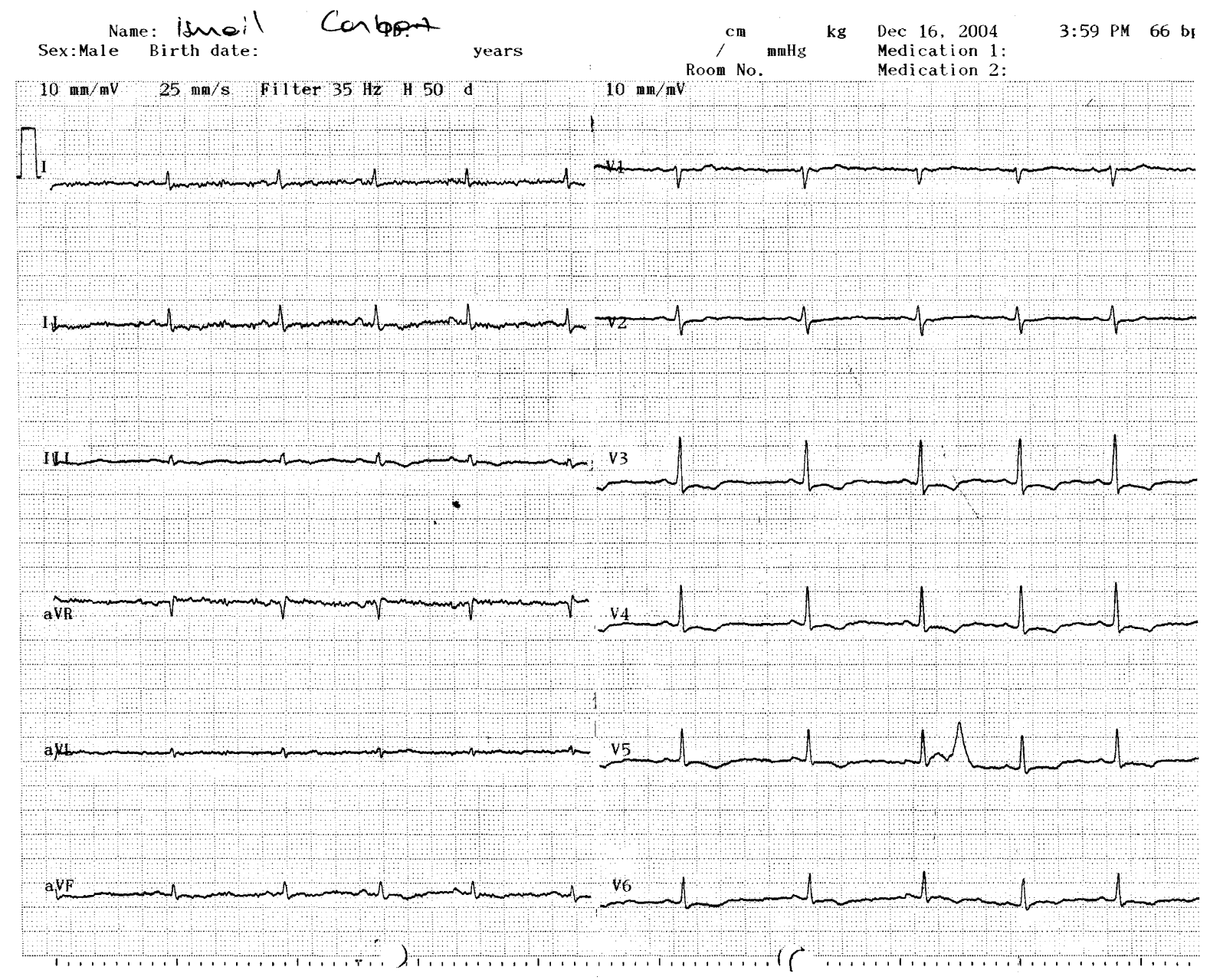

Figure 1. Electrocardiograpy of the patient showing $\mathrm{T}$ wave inversions in $\mathrm{V}_{2}-\mathrm{V}_{6}$ and $\mathrm{D}_{2}, \mathrm{D}_{3}$, aVF leads during admission. 


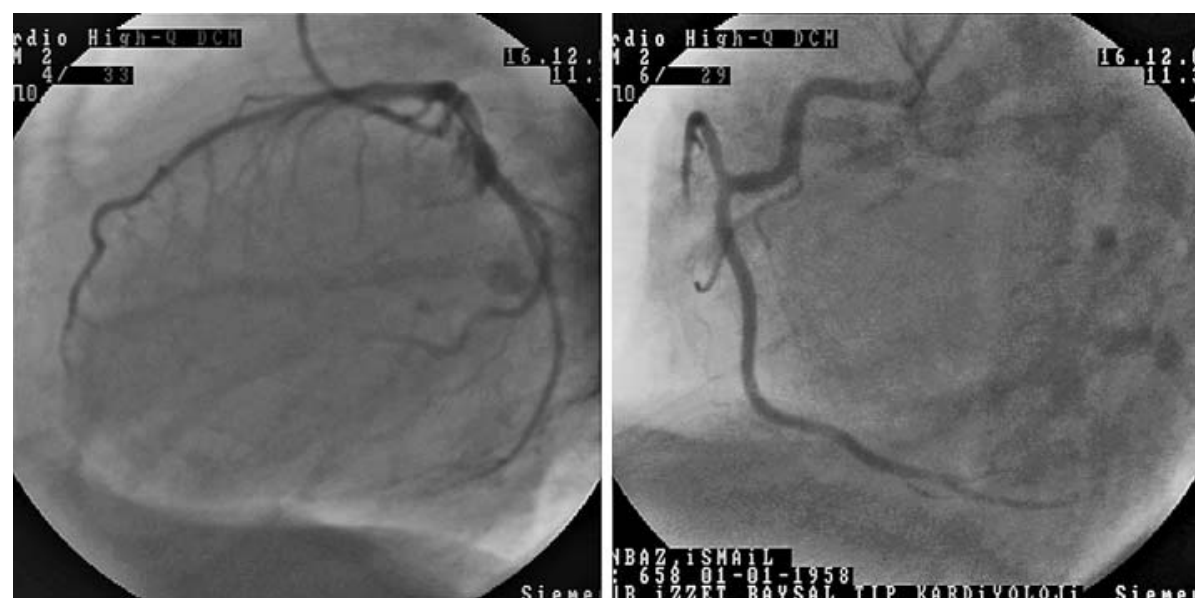

Figure 2. Coronary angiography of the patient showing no abnormalities and no atherosclerotic lesions (a) left coronary arteries; (b) right coronary artery.

initial stabilization, he underwent cardiac catheterization and coronary angiography. There was no atherosclerotic lesion in coronary arteries which may cause acute coronary syndrome in coronary angiography (Figure 2) and ergonovine provocation test was performed to exclude Prinzmetal' $s$ angina but the result was normal.

In view of the effort related dyspnea, weakness and elevated CK, CK-MB, abnormal ECG findings and normal coronary angiography; we further evaluated the patient for hypothyroidism. Thyroid function tests revealed a thyroid-stimulating hormone $(\mathrm{TSH})>75 \mathrm{mU} / 1$ (reference range $0.4-4.0 \mathrm{mU} / \mathrm{l}$ ), free thyroxin (fT4) $<0.30 \mathrm{ng} / \mathrm{dl}$ (reference range $0.8-1.9 \mathrm{ng} / \mathrm{dl}$ ), positive thyroid auto-antibodies, and thyroid ultrasonography showing mild diffuse hypertrophy.

Then, thyroxin therapy was started at a low initial dose of $25 \mu \mathrm{g}$, and was increased gradually. Two months later, the patient was completely symptom free and had a normal electrocardiography (Figure 3) and biochemical findings.

\section{Discussion}

Hypothyroidism affects approximately $1 \%$ of the general population [3]. In the absence of previous use of radioactive iodine or surgical treatment of
Graves' disease, this condition is usually caused by autoimmune mediated atrophy of the thyroid gland, or Hashimato's thyroiditis characterized by diffuse firm thyroid enlargement [4].

Hypothyroidism has some cardiovascular consequences, and the cardiac manifestations of hypothyroidism include sinus bradycardia, pericardial effusion, heart failure, and coronary atherosclerosis [4]. ECG changes including T-wave flattening or inversion, small $\mathrm{P}$ waves, a prolonged QT interval, heart block, intraventricular conduction defects, ventricular extra systoles and low QRS voltage have been also reported in patients with hypothyroidism [5].

Two different mechanisms have been proposed for the relationship between hypothyroidism and coronary artery disease. First, metabolic and hemodynamic changes in hypothyroid patients such as hypercholesterolemia and hypertension increase the risk of atherogenesis. Second, hypothyroidism creates a negative chronotropic and inotropic state diminishing myocardial oxygen demand, and may provoke underlying coronary ischemia [6].

In addition, hypothyroid patients have increased concentrations of creatinine kinase that is mostly due to increased CK-MM. However, CK-MB has also been reported to increase above reference values in hypothyroid patients without apparent myocardial damage [2]. This may create confusion 


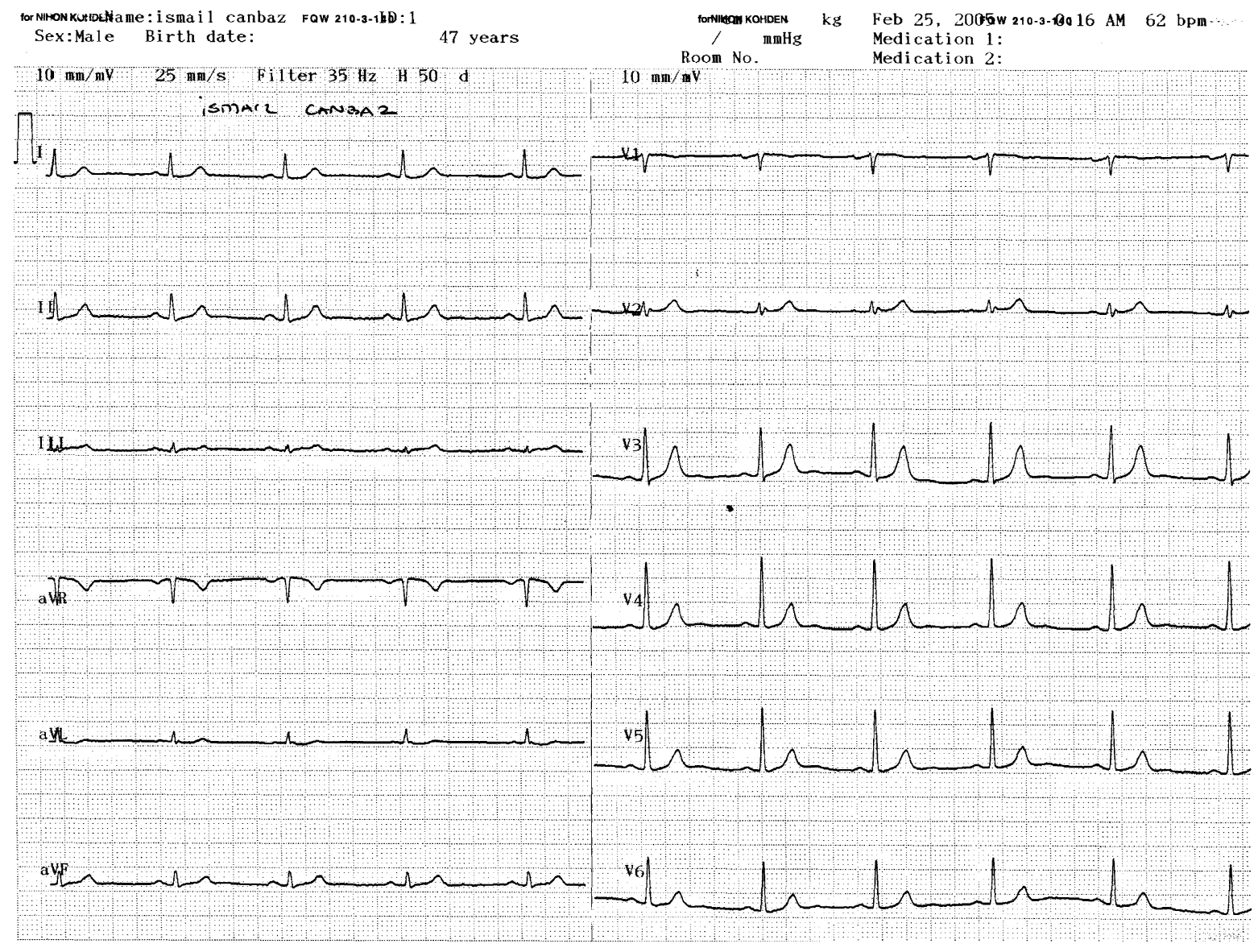

Figure 3. ECG of the patient taken 2 months later showing completely resolved $\mathrm{T}$ wave inversions with thyroxine treatment.

during the evaluation of myocardial injury in a hypothyroid patient presenting with chest pain. Troponin I is considered as a superior marker for the diagnosis of myocardial infarction, because it is found only in heart muscle [7]. Cohen et al. [7] reported that the level of troponin I did not increase in hypothyroid patients, and they suggested that its level should be investigated in case of an increase of CK and CK-MB levels if there is a high clinical suspicion [8]. On the other hand, there were some reports showing an increase in the level of troponin I without any myocardial damage in hypothyroid patients as in our case [9, 10]. Furthermore, most studies evaluating the cardiac troponin I as a marker for myocardial injury have been limited to the patients with cardiac, renal, or muscular disorders [9].
Consequently, the diagnosis of hypothyroidism presenting with classical symptoms and signs is easy. However, even in the absence of characteristic features, the diagnosis of hypothyroidism should be considered during the initial evaluation of patients presenting with symptoms of ischemic heart disease with raised $\mathrm{CK}, \mathrm{CK}-\mathrm{MB}$, troponins and/or ECG abnormalities. In addition, although it is suggested that troponin I should be measured for the diagnosis of myocardial infarction in patients with hypothyroidism, it should be kept in mind that troponin I might be increased without any myocardial damage in patients with hypothyroidism. Thus, coronary angiography might be considered as in our case due to the high incidence of coronary artery disease in this population and the presence of controversial findings about troponins. 


\section{References}

1. Klein I, Ojamaa K. Thyroid hormone and the cardiovascular system. New Engl J Med 2001; 344: 501-509.

2. Chan KM, Ladenson JH. Increased creatine kinase MB in the absence of acute myocardial infarction. Clin Chem 1986; 32: 2044-2051.

3. Gammage M, Franklyn J. Hypothyroidism, thyroxine treatment, and the heart. Heart 1997; 77: 189-190.

4. Toft AD, Boon NA. Thyroid disease and the heart. Heart 2000; 84: 455-460.

5. Slovis C, Jenkins R. ABC of clinical electrocardiography: Conditions not primarily affecting the heart. Br Med J 2002; 324: 1320-1323.

6. Kahaly GJ, Dillmann WH. Thyroıd hormone action in the heart. Endocr Rev 2005; 4: 1-70.

7. Cohen LF, Mohabeer AJ, Keffer JH, Jialal I. Troponin I in hypothyroidism. Clin Chem 1996; 42: 1494-1495.
8. Strachan SR, Afolabi O, Brown N, Gray D. Chest pain, enzymes and hypothyroidism. Postgrad Med J 2000; 76: $168-169$.

9. Khan IA, Tun A, Wattanasauwan N, Win MT, Hla TA, Hussain A, Vasavada BC, Sacchi TJ. Elevation of serum cardiac troponin I in noncardiac and cardiac diseases other than acute coronary syndromes. Am J Emerg Med 1999; 17: 225-229.

10. Grewal J, Gin K. Troponin. Marks the spot!. Perspect Cardiol 2004; 20: 35-40.

Address for correspondence: Huseyin Gunduz, MD, Department of Internal Medicine and Cardiology, Izzet Baysal Medical Faculty, Izzet Baysal University, Bolu, 14100 Turkey

Tel.: + 90-374-217-65-20

E-mail: drhuseyingunduz@yahoo.com 